\title{
LA COMUNICACIÓN NO VERBAL EN LA ENSEÑANZA DE INGLÉS COMO SEGUNDA LENGUA
}

\author{
Gloria Álvarez Benito \\ Universidad de Sevilla
}

\begin{abstract}
In this paper we focus on the lack of attention paid to nonverbal communication in second language teaching and learning. Communication is more than just what we say, it is also how we say it, and how we move when we say it. If native speakers use not only verbal, but also paralinguistic and kinesic mechanisms when communicating in their mother language, then, the three levels should be accounted for in second language teaching. Although some components of nonverbal communication may be universal, independent of culture, most of them are culturespecific and should be learned and acquired in the same way as we learn vocabulary or grammatical structures.
\end{abstract}

\section{OBjetivo}

Este artículo tiene como objetivo analizar la relevancia que tienen los elementos no verbales en el acto comunicativo para, posteriormente, valorar la necesidad de su inclusión en la enseñanza/aprendizaje de segundas lenguas.

Resulta paradójico que una parte tan importante de la comunicación no esté incluida, por lo general, en el marco de la enseñanza de segundas lenguas. En este estudio, pretendemos demostrar que la inclusión de la kinésica y el paralenguaje en los programas de segundas lenguas irá en beneficio de la comunicación, ya que mejorará enormemente la fluidez del hablante.

\section{INTRODUCCIÓN}

Cuando nos comunicamos, hacemos uso de diferentes instrumentos para codificar el mensaje que deseamos emitir. Por un lado, utilizamos elementos verbales, tanto a nivel oral como escrito, de acuerdo con unas reglas que varían dependiendo del idioma. Por otro lado, utilizamos elementos no verbales que van desde los movimientos y posturas que adoptamos hasta los sonidos que emitimos que no constituyen palabras. Estos recursos no verbales son conocidos, respectivamente, como kinésica y paralenguaje.

Dependiendo del mensaje en cuestión que estemos transmitiendo o interpretando, habrá casos en los que el mayor peso de la comunicación recaiga sobre elementos verbales, mientras que en otros casos lo haga sobre elementos no verbales, y en otras ocasiones ese peso estará compartido entre la comunicación verbal y la comunicación no verbal. Por lo tanto, sería erróneo afirmar, a priori, que la comunicación verbal es más importante que la no verbal. 
Debido al papel secundario al que ha sido habitualmente relegada la comunicación no verbal (en adelante CNV), es poco común que los elementos kinésicos y paralingüísticos estén contemplados en la enseñanza de segundas lenguas, hecho que se puede constatar fácilmente con una simple lectura de los programas o índices de contenidos de diferentes cursos de idiomas. Esta grave omisión hace que los estudiantes de segundas lenguas, en líneas generales, se preocupen sólo por transmitir correctamente lo verbal, mientras que los elementos de CNV son trasladados directamente de una lengua (la lengua materna) a otra (la segunda lengua). En la mayoría de las ocasiones, el estudiante ni siquiera es consciente de que está cometiendo un error, pues piensa que la CNV es universal.

Es cierto que existen algunos componentes no verbales que podríamos considerar de carácter universal, básicamente los relacionados con gestos o kinésica facial y que implican emociones o estados de ánimo, pero eso no es lo habitual. Por el contrario, los elementos no verbales suelen estar condicionados por una cultura y deben ser adquiridos y aprendidos del mismo modo en el que aprendemos el vocabulario o las estructuras gramaticales de una segunda lengua.

No es extraño que un estudiante español diga correctamente en inglés 'nos fuimos del pub porque estaba abarrotado de gente' (we left the pub because it was crowded) $\mathrm{y}$, sin embargo, utilice a la vez el signo kinésico español en lugar del inglés. Si hace esto, la kinésica no sólo no servirá como refuerzo de la comunicación verbal, sino que, por el contrario, estaremos distorsionando el mensaje emitido, ya que nuestro receptor no entenderá qué tiene que ver nuestra kinésica con lo que estamos diciendo:

Figura 1

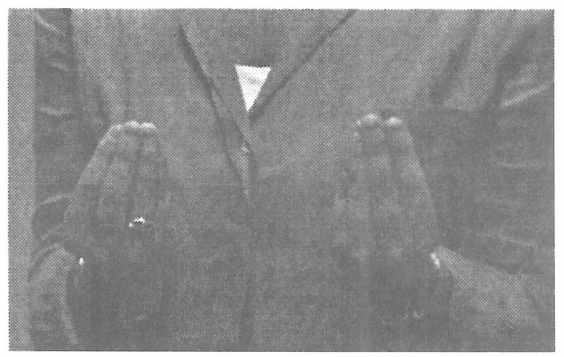

Figura 2

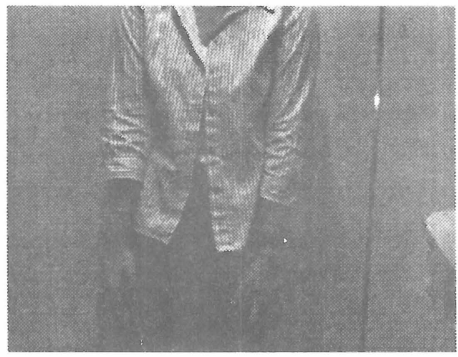

La figura 1 muestra la kinésica empleada en español para decir "está lleno/abarrotado", mientras que la figura 2 muestra la kinésica utilizada en inglés. Está claro que la una y la otra difieren notablemente. La figura 1 expresa el proceso por medio del cual un lugar se llena, mientras que la figura 2 muestra el resultado final.

Pero estos errores que surgen del mal uso de la kinésica, y que podríamos considerar falsos amigos kinésicos, son también extensibles al paralenguaje. Ningún estudiante dudaría de que el inglés y el español emplean vocablos distintos para expresar las acciones de los sonidos emitidos por animales. Es decir, en español decimos ladrar, mientras que en inglés decimos bark; o en español cacarear y en inglés cluck; o rebuznar frente a bray. Pero esto no implica que el estudiante sepa que el paralenguaje de esos sonidos sea diferente. Es decir, a un estudiante español, por ejemplo, le cuesta trabajo asimilar que un gallo diga en español quiquiriquí y que en inglés diga cock-a-doodle-doo. Esto se debe a que, si es cierto que estos elementos quasiléxicos imitan el sonido que sucede en la 
realidad, nos extraña que una cultura y otra no tengan la misma interpretación de una misma realidad, es decir, nos sorprende que ingleses y españoles perciban una misma realidad de manera distinta.

Dentro del nivel paralingüístico, habría también que tener en cuenta otros elementos no verbales como la entonación, el volumen, el timbre, etc., que en inglés y español difieren bastante.

Afortunadamente, cada vez son más los lingüistas que reconocen el papel de la $\mathrm{CNV}$, no como algo meramente anecdótico, sino como constituyente fundamental del acto comunicativo. Así, al respecto, señala Poyatos (1975) "What has hitherto been disregarded by linguists in the vocal output of people has as much structural and functional claim to be incorporated into language as words do."

Prueba de la importancia que tienen los elementos no verbales en la comunicación es la proliferación de los denominados smileys en los correos electrónicos y en los mensajes de texto. Este fenómeno hace que la CNV no sólo sea una característica típica o exclusiva del lenguaje oral, sino que se hace extensiva al lenguaje escrito. Algunos de estos smileys representan simplemente la kinésica facial típica de estados de ánimo, como los que mostramos a continuación:

:-) , : - D , ambos para indicar alegría; :- ( , (:-), ambos expresando tristeza.

Otros representan movimientos faciales o del cuerpo, como:

$$
\text { [], que significa 'abrazo' (hug); ;-), para guiñar (wink). }
$$

Es obvio que una enseñanza de L2 centrada sólo en los elementos verbales traerá consigo una comunicación artificial, limitada al contexto del aula, e incompleta, ya que los estudiantes no podrán cooperar de manera activa en el proceso comunicativo ni como emisores ni receptores.

\section{Metodología del estudio}

Para estudiar la relevancia de los elementos no verbales, tanto paralingüísticos como kinésicos, en la enseñanza y aprendizaje de inglés como L2, llevamos a cabo un experimento.

El primer paso en este experimento consistió en la elaboración de una encuesta a la que, posteriormente, serían sometidos nuestros informantes. Como veremos a continuación, los objetivos de cada uno de los apartados de la encuesta difieren notablemente. Así, hay enunciados encaminados a una respuesta de producción kinésica. Otros, sin embargo, pretenden forzar al informante a que utilice determinados recursos paralingüísticos. En otras ocasiones, nuestro interés principal será analizar la manera en la que los mecanismos no verbales interactúan con los verbales.

Como podemos apreciar más adelante, parte de la encuesta se realiza en español, que es la lengua materna de los informantes de nuestro experimento, y la otra parte se realiza en inglés, que es la lengua que los informantes están aprendiendo, y por lo tanto, su segunda lengua. El motivo de esta combinación de español e inglés es facilitar el posterior contraste y análisis de la CNV codificada por el mismo informante en la lengua materna, L1, y en la lengua destino, L2. 


\section{Encuesta}

\section{Sección en español}

1. Se le pide al informante que describa lo que ve en una fotografía que le acabamos de entregar. Ésta muestra la pista de baile de una discoteca abarrotada de gente joven que se encuentra bailando.

2. ¿Qué diferencias encuentras entre el sonido producido por una trompeta y el producido por una flauta?

3. ¿Cómo explicarías en qué consiste una Montaña Rusa a alguien que nunca ha visto una?

4. ¿Has estado alguna vez en una situación en la que le pasa algo desagradable a alguien y a ti te entra la risa?

5. ¿Podrías describir la situación más bochornosa para ti en la que habrías querido que te tragara la tierra?

\section{Sección en inglés}

1. Do you like the Holy Week in Seville? Why or why not? What do you do during that week?

2. How would you describe a steam train?.

3. Your five-year old niece or nephew is visiting a farm in a few days. Give her/him a detailed description of the animals and things she/he is going to find there.

4. Have you ever been to a concert? Tell me about your experience there and describe what you saw.

5. How would you describe a Merry-go-round to a child who has never seen one?

El segundo paso de nuestro experimento fue la selección de informantes: 60 hablantes nativos de español que estudian inglés como segunda lengua. Para seleccionar estos informantes se tuvieron en cuenta, principalmente, dos criterios: nivel de conocimiento de la segunda lengua y sexo. Así, los 60 informantes se dividían en grupos de la siguiente manera:

10 varones de nivel principiante.

10 mujeres de nivel principiante.

10 varones de nivel intermedio.

10 mujeres de nivel intermedio. 
$>10$ varones de nivel avanzado.

$>10$ mujeres de nivel avanzado.

La razón de esta clasificación era poder determinar, en primer lugar, hasta qué punto el nivel de fluidez verbal era equiparable al nivel de fluidez no verbal y, en segundo lugar, analizar si el nivel de fluidez verbal del informante en la L2 podría condicionar el uso de mecanismos no verbales en la comunicación. Además, queríamos comprobar si las diferencias de sexo daban, como consecuencia, resultados diferentes o afines.

\section{ANÁlisis de Resultados}

Tras un análisis y valoración de la información codificada por los informantes en las encuestas realizadas, lo primero que podemos comprobar es que se utilizan muchos más mecanismos de comunicación no verbal que de comunicación verbal para transmitir el mensaje. Estos resultados son acordes con la teoría de Albert Mehrabian (1972), según el cual comunicamos mucho más por medio de la kinésica y del paralenguaje que por medio de elementos verbales:

$7 \%$ a través de elementos verbales.

$38 \%$ por medio de paralenguaje.

$55 \%$ por la kinésica.

En el gráfico siguiente (Figura 3) se exponen los porcentajes de cada uno de estos mecanismos (comunicación verbal, paralenguaje y kinésica) codificados por nuestros informantes:

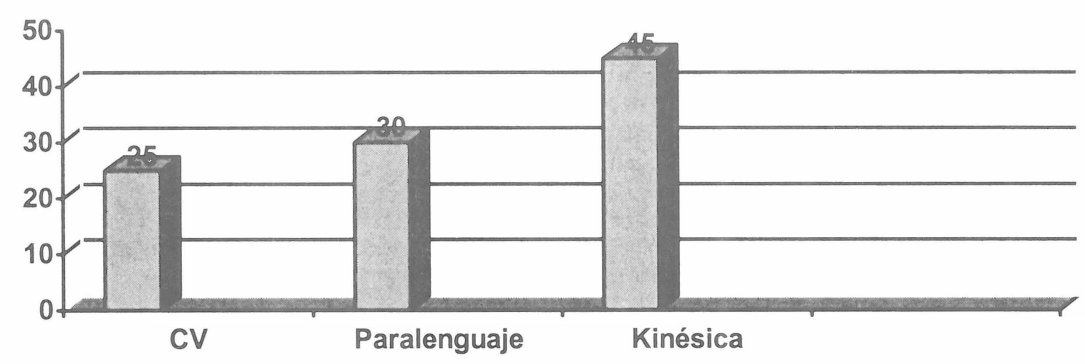

Figura 3

Teniendo en cuenta estos porcentajes, está claro que lo que decimos importa bastante menos que cómo lo decimos o cómo actuamos. Nuestros informantes utilizaron un $75 \%$ de recursos de CNV (paralenguaje y kinésica) para transmitir sus mensajes, frente al 25\% de comunicación verbal.

Otro aspecto importante que resulta de nuestro experimento es la diferencia que existe entre hombres y mujeres en lo que respecta a comportamiento no verbal. Según nuestros resultados, los informantes mujeres utilizan una kinésica más abundante y notoria que la de los hombres (tanto en la L1 como en la L2), como se muestra en la Figura 4. Por el 
contrario, hemos podido apreciar que los hombres utilizan más mecanismos paralingüísticos que las mujeres, también en las dos lenguas:

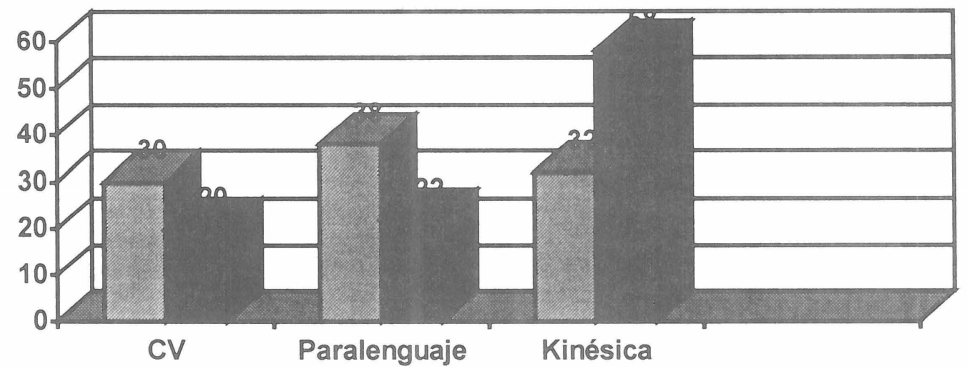

\section{[ Hombres \\ Mujeres}

Figura 4

Como podemos ver en esta Figura 4, los valores de codificación de paralenguaje y kinésica de hombres y mujeres están bien diferenciados. Resulta curioso que la diferencia de porcentaje que hay entre la comunicación verbal y la kinésica en los hombres sea la misma que la que encontramos entre la comunicación verbal y el paralenguaje en las mujeres. Obviamente, estos resultados están condicionados por el tipo de preguntas que hemos realizado a nuestros informantes. En un entorno natural, probablemente, los porcentajes de recursos paralingüísticos no serían tan altos. Sin embargo, lo que nos interesa destacar es que ante el mismo tipo de situación, hombres y mujeres han tenido una respuesta bien distinta.

También hemos podido comprobar en este experimento que nuestros informantes codifican más mecanismos no verbales en la L2 que en la L1 (Figura 5):
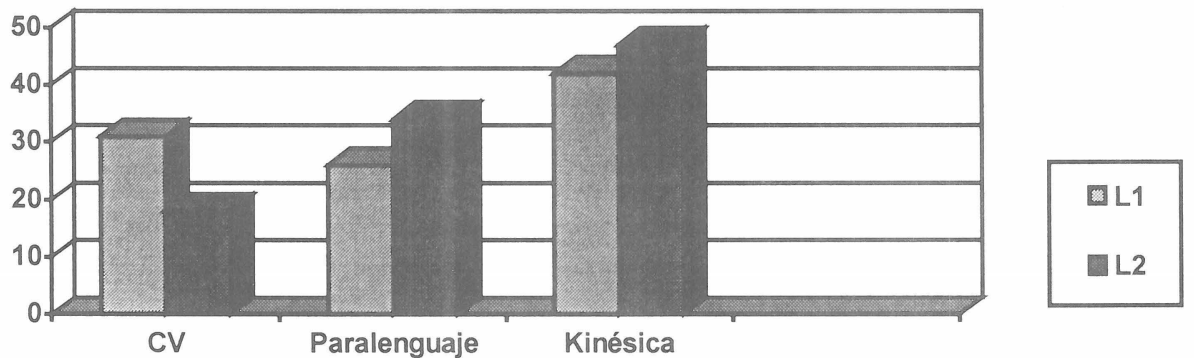

Figura 5

Este gráfico muestra cómo el porcentaje de comunicación verbal se reduce considerablemente en la L2 y, como contrapartida, aumentan los niveles de recursos paralingüísticos y kinésicos. Estos resultados vienen a confirmar la importancia que tiene un entrenamiento previo en la CNV de la L2. En primer lugar, los informantes deberían 
saber que no todas las lenguas hacen el mismo uso de la CNV. Se ha distinguido entre lenguas frías y lenguas cálidas y el inglés se encuentra entre las primeras, mientras que el español se encuentra entre las segundas. Por lo tanto, cabría esperar que la codificación de $\mathrm{CNV}$ en inglés fuera menor que la que encontramos en español, ya que los hablantes de lengua inglesa utilizan muchos menos recursos no verbales que los españoles en el acto comunicativo.

Pero obviamente, el problema de estos resultados no está sólo en la cantidad de CNV codificada en la L2, sino que, además, muchos de esos elementos de CNV que nuestros informantes han codificado en la L2 son falsos amigos kinésicos o paralingüísticos. Por ejemplo, casi todos los informantes han utilizado el falso amigo kinésico que mostrábamos en la figura 1 para expresar que un lugar está abarrotado de gente (generalmente, en las respuestas de las preguntas 1 y 4 de la sección de inglés). En cuanto a los falsos amigos paralingüísticos, podemos decir que han sido la tónica general en la respuesta a la pregunta 3 de la sección de inglés (para describir los sonidos producidos por los animales).

Si distinguimos por sexos y diferenciamos los valores entre la L1 y la L2, obtenemos los siguientes resultados (Figura 6):

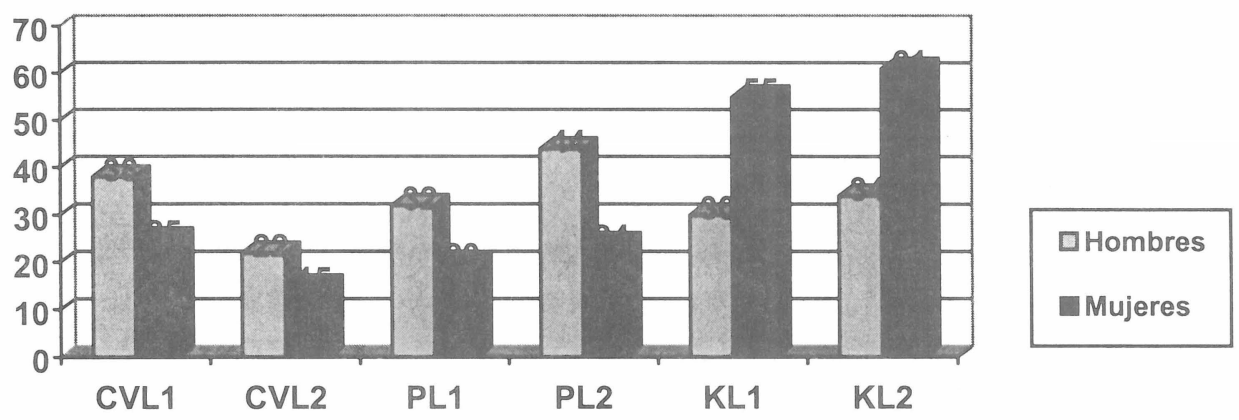

Figura 6

Tal y como mencionábamos con anterioridad, la razón de estos resultados pudiera estar en la falta de competencia discursiva que tiene el informante en la L2. Aunque queda patente que la codificación de recursos no verbales es también amplia en la L1, en la L2 su producción está directamente relacionada con el nivel de fluidez del informante. A menor fluidez verbal, mayor uso de mecanismos no verbales. Podemos afirmar que este comportamiento se debe a la necesidad del hablante de suplir las carencias que tiene en su comunicación en la L2 con mecanismos con los que él supuestamente se siente más familiarizado.

Los gráficos siguientes muestran la producción no verbal de los informantes en la L1 y en la L2, según su nivel de fluidez en la segunda lengua, en este caso en inglés. Aunque la producción de CNV en la L2 supera en todos los casos a la L1, veremos cómo su porcentaje irá decreciendo a medida que aumenta el nivel de fluidez del informante en la lengua inglesa.

La Figura 7 corresponde a los valores obtenidos de los veinte informantes del nivel principiante. En consecuencia, este gráfico indica los valores de los estudiantes con el nivel de fluidez más bajo en la L2: 


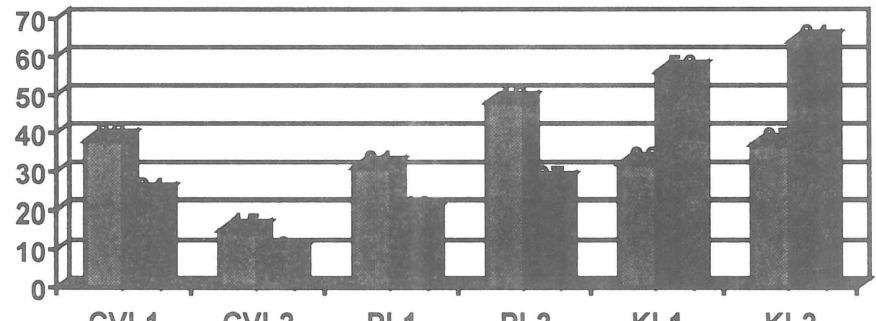

Hombres

Mujeres

$\begin{array}{llllll}\text { CVL1 } & \text { CVL2 } & \text { PL1 } & \text { PL2 } & \text { KL1 } & \text { KL2 }\end{array}$

Figura 7

La Figura 8 corresponde a los resultados de los informantes de nivel intermedio. Aquí podemos apreciar cómo los valores obtenidos en comunicación verbal de la L2 superan ligeramente los valores del nivel principiante, mientras que los porcentajes de paralenguaje y kinésica descienden:

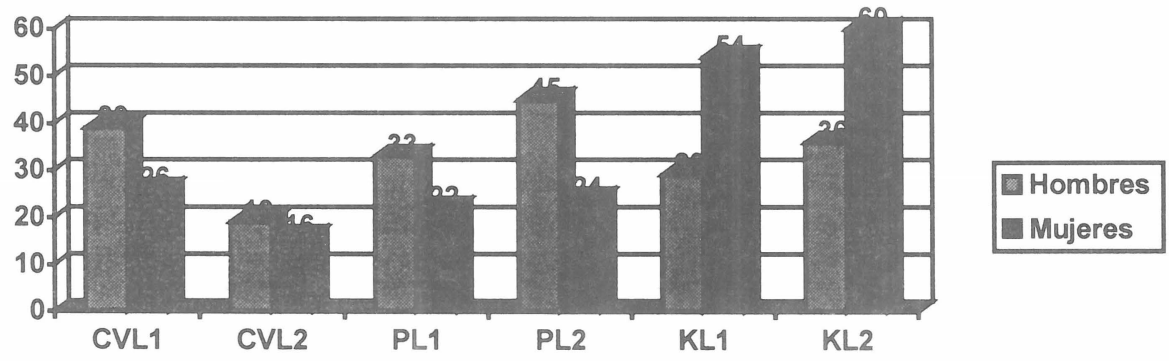

Figura 8

Por último, la Figura 9 muestra los resultados de los informantes del nivel avanzado, donde podemos comprobar que mientras los porcentajes de CNV de la L1 se mantienen, los de la L2 siguen descendiendo: 


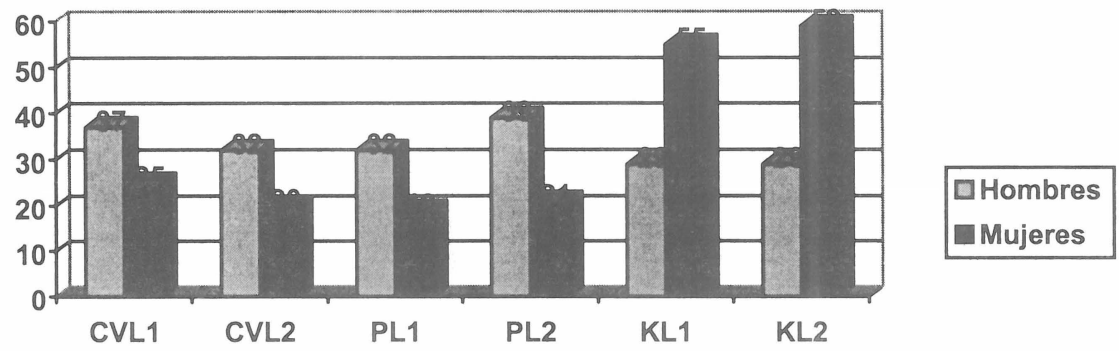

Figura 9

A continuación, y para tener una visión general de cómo cambian los porcentajes de CNV en la L2 mientras que en la L1 se mantienen, mostramos dos gráficos con todos los valores. La siguiente figura (Figura 10) muestra los datos de los hombres:

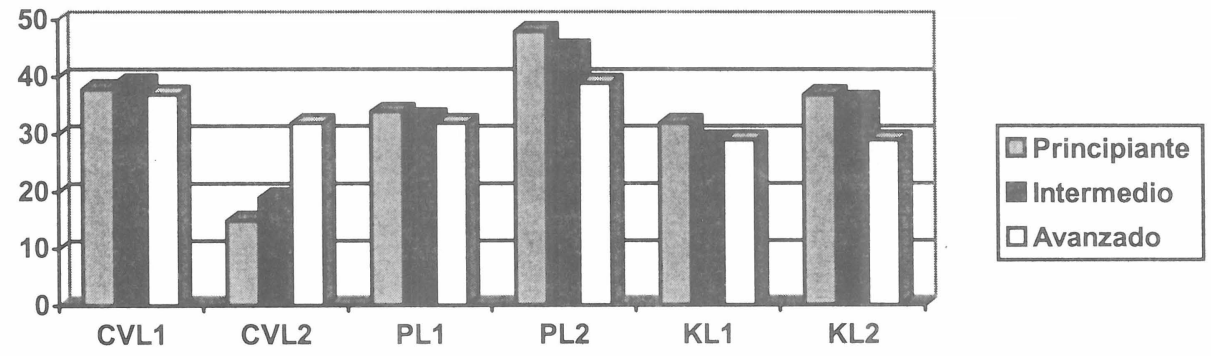

Figura 10

La Figura 11 muestra los resultados de las mujeres: 


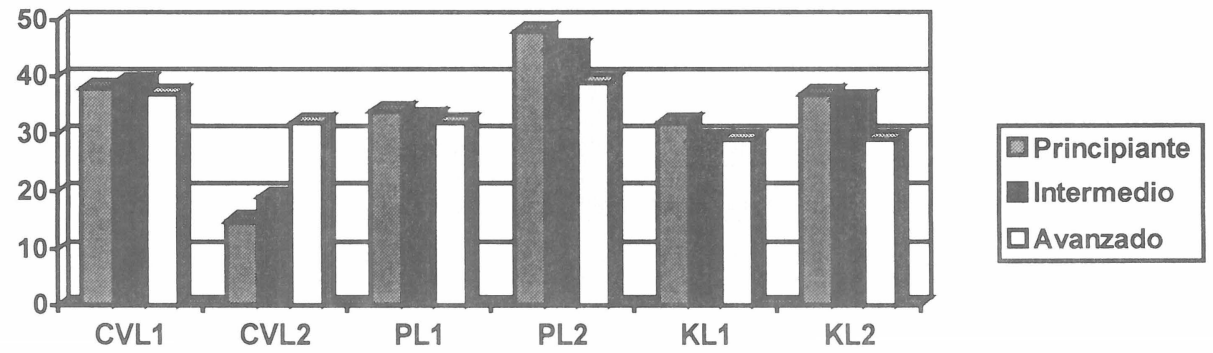

Figura 10

También se han podido comprobar diferencias notables en lo que respecta a la función que desempeñan los elementos no verbales codificados en la L1 y en la L2.

Así, en la L2 se utiliza un mayor número de ilustradores, tanto por mujeres como por hombres. Sabemos que la función de los ilustradores es reforzar de manera no verbal lo que se expresa verbalmente. La diferencia en el uso de este tipo de elementos entre la L1 y la L2 es mayor cuanto menor es el nivel de los informantes. Es fácil pensar que la razón de este mayor uso en la L2 se debe a que el informante duda de su capacidad lingüística para hacerse entender y, por eso, hace uso de lo no verbal como recurso de refuerzo, por si el mensaje verbal no ha sido lo suficientemente claro.

Si en el punto anterior comentábamos el aumento del uso de ilustradores en la L2, el incremento de los denominados emblemas es aún mayor. En cierta medida, este resultado es el que esperábamos, ya que los emblemas son elementos no verbales que sustituyen la comunicación verbal. Es decir, si el informante carece de los conocimientos verbales para expresar un determinado mensaje en la L2, entonces se va a ver obligado a recurrir a otros mecanismos (en este caso, no verbales) para codificar ese mismo mensaje. Al igual que sucedía en el punto anterior, cuanto menor es el nivel de los informantes mayor es el uso de este tipo de mecanismos. El uso de emblemas ha sido un recurso muy extendido entre nuestros informantes en los casos de expresión de movimiento, dirección, sonidos emitidos por máquinas, o sonidos emitidos por animales.

Curiosamente los casos de elementos no verbales cuya función es reguladora han aparecido principalmente en la L1. Algunos informantes daban pequeños golpecitos en la mesa, bien con la mano o con un bolígrafo, a la vez que realizaban una descripción; otros se balanceaban al hablar, moviendo el torso hacia delante y hacia atrás; otros llevaban el ritmo de la conversación con pequeños movimientos del dedo índice, de la mano derecha, o bien con el pie, pero siempre durante las respuestas realizadas en español, la L1. Una de las explicaciones que podemos dar al hecho de que los elementos reguladores tengan una representación mínima en la L2 es que el emisor está mucho más preocupado por el contenido de su mensaje y por hacerse entender que por su ritmo o estructura, que se convierten en algo secundario. 
En lo que respecta a la CNV con función de expresión de emociones, existe un equilibrio entre la L1 y la L2. Quizá se deba a que la expresión de muchos de estos gestos se producen de manera no controlada

Otro aspecto a destacar es que en la L1 se han encontrado algunos usos de mecanismos no verbales que contradicen lo expresado verbalmente, generalmente con una función irónica, sin embargo, no hemos hallado ningún caso en la L2.

A tenor de los resultados obtenidos en este experimento sería conveniente que nos hiciéramos un replanteamiento de los programas curriculares de enseñanza de lenguas extranjeras. En este sentido, deberíamos plantearnos por qué motivo no entregamos a nuestros estudiantes algunos inventarios kinésicos y paralingüísticos, del mismo modo que les ofrecemos listados de vocabulario. Si la comunicación verbal y la no verbal no se suelen dar de manera aislada, sino que interactúan, su aprendizaje debería ser también al unísono.

\section{ConClusiones}

A lo largo de este estudio se han podido comprobar distintos aspectos en relación a la comunicación no verbal que resumimos a continuación.

En primer lugar, un gran porcentaje de la información que comunicamos se codifica a través de mecanismos no verbales, bien kinésicos, bien paralingüísticos.

En segundo lugar, la codificación de los elementos de comunicación no verbal no se rige por unas reglas universales que se puedan aplicar a todas las lenguas. Por el contrario, la comunicación no verbal es característica de una cultura determinada y por lo tanto su significado y uso no responde a un estímulo innato del ser humano, sino que se aprende igual que aprendemos a utilizar las herramientas de la comunicación verbal.

También hemos podido confirmar que un mismo informante hace un uso mayor de la CNV en la L2 que en la L1. Esto es debido a la necesidad que siente el hablante de suplir su falta de fluidez verbal con elementos no verbales que él considera, erróneamente, universales. En este sentido hay que puntualizar que la codificación no verbal de las mujeres en la L2 supera en cantidad a la de los hombres, pero esto se hace también extensivo a la L1. Lo que sí parece representativo es que tanto en mujeres como en hombres se da el mismo porcentaje de aumento en la producción no verbal en la L2. Obviamente este incremento de CNV en la L2 depende del nivel de producción y expresión del informante.

La fluidez de los informantes en comunicación verbal no se corresponde con su fluidez en comunicación no verbal. Es decir, la fluidez de comunicación no verbal es siempre menor que la fluidez a nivel verbal.

Teniendo en cuenta todos estos factores, podemos concluir que la inclusión de la comunicación no verbal en la enseñanza de segundas lenguas se hace no sólo aconsejable, sino más bien imprescindible. Así, se conseguirá, por un lado, mejorar la fluidez del hablante en la L2, tanto a la hora de codificar un mensaje en la segunda lengua como al decodificarlo o interpretarlo. Por otro lado, y siguiendo los resultados obtenidos en nuestro estudio, el hablante no hará un uso desbordado de elementos de comunicación no verbal en su afán de suplir su falta de fluidez verbal, ya que será consciente de que no puede hacerlo de manera indiscriminada al no ser universal.

Por último, la inclusión de la comunicación no verbal en la enseñanza de segundas lenguas favorece la naturalidad en la comunicación. Cuando una persona se comunica haciendo uso de elementos verbales exclusivamente, resulta demasiado rígida y, en 
consecuencia, poco natural. $\mathrm{Si}$, por el contrario, una persona se comunica abusando de los elementos no verbales y haciendo poco uso de los recursos verbales puede llegar a resultar ridículo y, en algunas ocasiones, muy poco apropiado. Por ese motivo, lo que resulta más natural es la combinación y coordinación de ambos recursos, haciendo uso de ellos en el momento oportuno y de la manera más adecuada. Para saber codificar los elementos verbales y los no verbales en armonía y de manera correcta es necesario que su adquisición se produzca de manera simultánea e interactiva.

\section{BIBLIOGRAFÍA}

POYATOS, F., "Cross cultural analysis of paralinguistic 'alternants' in face-to-face interaction" en A. Kendon, R. Harris and M.R. Key, Organization of Behavior in Faceto-Face Interaction (The Hague 1975) 285-314.

MEHRABIAN, A., Nonverbal Communication (Chicago 1972) 182. 